Reprod. Nutr. Dévelop., 1981, 21 (3), 421-428.

\title{
Effet du jeûne sur les principaux métabolites sanguins et hépatiques de la néoglucogenèse et de la cetogenèse chez la caille domestique adulte (Coturnix coturnix japonica)
}

\author{
par R. DIDIER, C. RÉMÉSY *, C. DEMIGNÉ * \\ Laboratoire de Biologie animale, E.R.A. 408 \\ Université Clermont II, BP 45, 63170 Aubière, France. \\ * Laboratoire des Maladies méfaboliques, I.N.R.A., \\ Theix, 63110 Beaumont, France.
}

Summary. Effect of starvation on the main plasma and liver metabolites of gluconeogenesis and ketogenesis in the adult domestic quail (Coturnix coturnix japonica).

The changes in the main plasma and liver metabolites of gluconeogenesis and ketogenesis have been studied in quails during 72 -hr starvation. In those conditions, glycaemia barely decreased, whereas liver glycogen and plasma and liver lactate dropped, suggesting that lactate was utilized extensively for gluconeogenesis. In contrast, plasma alanine decreased only slightly. During starvation, plasma free fatty acids and cholesterol were significantly enhanced, whereas plasma triglycerides decreased. Ketogenesis seemed very active. 3-Hydroxybutyrate concentrations were markedly higher in the plasma than in the liver, indicating that mechanisms other than simple diffusion processes should be considered for hepatic ketone body release.

The present results suggest that metabolic response to prolonged starvation in the quail does not differ markedly from that observed in other birds. However, the utilization of glucose and ketone bodies by peripheral tissue and of some substrates, such as alanine or pyruvate, for gluconeogenesis must be further investigated.

Le métabolisme des lipides et des hydrates de carbone chez l'oiseau domestique diffère en certains points de celui des mammifères.

En ce qui concerne l'homéostasie glucidique, ces différences se situent, entre autres, au niveau de la glycémie qui est nettement plus élevée que celle des mammifères (Pearce, 1971), de l'activité et de la localisation de certaines enzymes impliquées dans la néoglucogenèse. Ainsi, la PEP carboxykinase est mitochondriale chez le poulet (Söling et al., 1973) alors qu'elle est localisée dans le cytosol chez le rat (Lardy, 1965) et qu'elle est à la fois mitochondriale et cytosolique chez l'homme (Söling et Kleineke, 1976). De plus, la nature des isoenzymes de la pyruvate kinase et la régulation de son activité sont très différentes de celles des mammifères (Pearce, 1977). Une autre différence importante se situe au niveau de la phosphorylation ou de la déphosphorylation du glucose puisque l'oiseau n'a pas de glucokinase ef que les concentra- 
tions en glucose-6-phosphate sont très élevées (O'Neill et Langslow, 1978). II existe aussi des particularités dans l'utilisation de certains précurseurs néoglucogéniques (Sarkar, 1971 ; Davison ef Langslow, 1975). De même, des divergences apparaissent dans le contrôle endocrinien de ces mécanismes (Langslow et Hales, 1971).

La plus grande partie des études effectuées chez les oiseaux a porté sur le poulet et le pigeon soumis au jeûne ou à différents régimes. Aussi, afin d'apporter de plus amples précisions sur ces phénomènes, nous avons étudié la réponse de la caille domestique après un jeûne de $72 \mathrm{~h}$.

\section{Matériels et méthodes.}

Nous avons utilisé des cailles de race Gérardmer, âgées de 12 semaines, d'un poids moyen de $200 \mathrm{~g}$. Les animaux mâles et femelles sonf logés dans des batteries métalliques dans une pièce à température de $20-22^{\circ} \mathrm{C}$ et répartis en deux lots. Dans le lot servant de témoin, les animaux sont nourris exclusivement d'un aliment granulé, l'eau de boisson étant, comme l'aliment, distribuée à volonté. Le deuxième lot esł constitué d'animaux mis à jeûn pendant $72 \mathrm{~h}$. La composition des granulés, à base de céréales et de tourteaux de soja, était la suivante : matières protéiques brutes : 18 p. 100 , matières grasses : 3 p. 100 , matières cellulosiques : 7 p. 100 et minéraux : $10 \mathrm{p}$. 100. Les animaux étaient soumis à un éclairage permanent de $5 \mathrm{~h}$ à $21 \mathrm{~h}$ (lumière nafurelle et artificielle).

Les animaux sont sacrifiés le matin entre $9 \mathrm{~h}$ et $10 \mathrm{~h}$, par décapitation, le sang étant recueilli dans des tubes héparinés. Une partie est déprotéinisée par $\mathrm{HClO}_{4} 0,6 \mathrm{M}$, tandis que le reste est centrifugé. Le plasma subit la même déprotéinisation. Une partie du surnageant acide est immédiatement neutralisée pour la détermination de l'acétoacétate. Le foie est prélevé à l'aide de clamps et plongé immédiatement dans l'azote liquide puis broyé dans $\mathrm{HClO}_{4}(1 \mathrm{~g}$ de tissu/7 $\mathrm{ml})$. Les différents métabolites sont dosés par des méthodes enzymatiques : glycogène (Keppler et Decker, 1974), lactate (Gutmann et Wahlefeld, 1974), glucose (Bergmeyer et al., 1974), alanine (Williamson, 1974), 3-hydroxybutyrate (Williamson et Mellanby, 1974), acétoacétate (Mellanby et Williamson, 1974), triglycérides (Wahlefeld, 1974), cholestérol (Röschlau ef al., 1974). Les acides gras non estérifiés sont dosés par la méthode de Fahlolt et al. (1973).

La concentration intracellulaire de différents métabolites est exprimée soit en umoles par gramme de foie, soit en $\mu$ moles par $\mathrm{ml}$ d'eau hépatique en tenant comple du fait que le poids sec du foie d'animaux nourris est $32 \mathrm{p} .100$ du poids frais et que celui des animaux à jeun esi de 29 p. 100.

Le test utilisé pour déterminer la signification statistique des moyennes était le test $t$ de Student.

\section{Résultats.}

Concentration de divers métabolites au niveau du sang ef du plasma (tabl. 1 et 2). - Un jê̂ne de 72 h se traduit, chez la caille adulte, par une baisse significative des concen- 
TABLEAU 1

Concentrations de différents métabolites au niveau du sang total

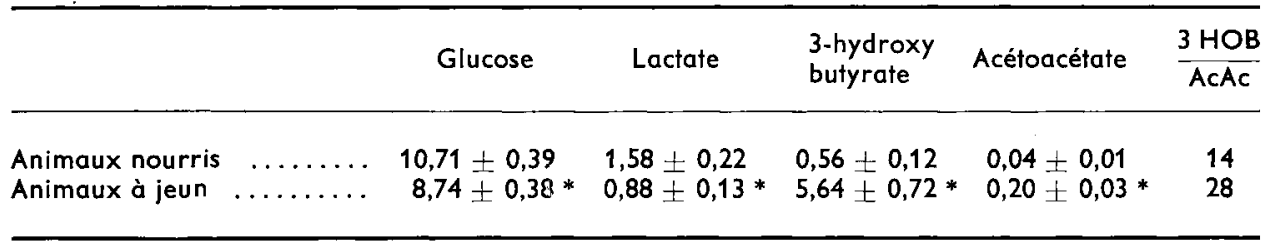

Les valeurs correspondent à la moyenne $\pm S E M$ pour 12 cailles exprimées en $\mu \mathrm{moles} / \mathrm{ml}$ de sang. La signification statistique pour $\mathrm{P}<0,05$ est indiquée par *.

\section{TABLEAU 2}

Concentrations plasmatiques de différents substrats de la néoglucogenèse et de la cétogenèse ainsi que des triglycérides et du cholestérol total

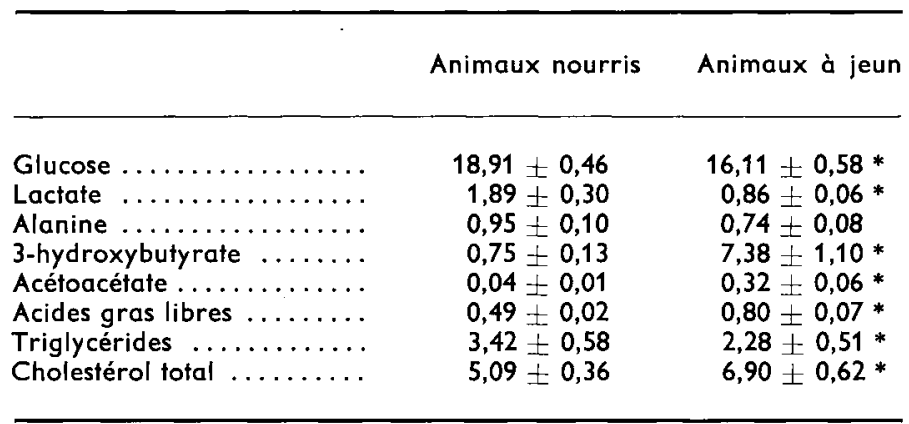

Les valeurs correspondent à la moyenne $\pm S E M$ pour 12 cailles exprimées en $\mu \mathrm{moles} / \mathrm{ml}$ de plasma. La signification statistique pour $\mathrm{P}<0,05$ est exprimée par *.

trations de glucose sanguin et plasmatique. Cette diminution de la glycémie est associée à une chute des concentrations de lactate. Chez l'oiseau, à la différence du rat, l'alanine chute modérément lors du jeûne, ce qui suggère que son utilisation hépatique est relativement réduite. Le jeûne provoque également une augmentation des concentrations en 3-hydroxybutyrate et en acétoacétate. Le rapport 3-hydroxybutyrate/acétoacétate, déjà élevé chez l'animal nourri, augmente encore chez l'animal à jeun.

L'augmentation des corps cétoniques sanguins est accompagnée d'une augmentation des concentrations d'acides gras libres et d'une baisse des triglycérides plasmatiques. A jeun, le cholestérol total s'élève significativement.

Concentration de divers métabolites au niveau du foie (tabl. 3 et 4). - Cet organe est le siège d'une glycogénolyse très prononcée et, après $72 \mathrm{~h}$ de jeûne, la concentration de glycogène a diminué de 86 p. 100. La concentration du lactate chute de façon importante alors que celle des corps cétoniques s'accroît notablement. A jeun, le rapport 3-hydroxybutyrate/acétoacétate est moins élevé dans le foie que dans le plasma.

Les concentrations en 3-hydroxybutyrate sont moins élevées dans l'eau hépatique que dans l'eau plasmatique. 
TABLEAU 3

Concentrations plasmatiques ef hépatiques des corps céfoniques

\begin{tabular}{|c|c|c|c|}
\hline & & 3-hydroxybutyrate & Acétoacétate \\
\hline Animaux nourris & $\begin{array}{l}\text { Plasma } \ldots \ldots \\
\text { Foie } \ldots . . .\end{array}$ & $\begin{array}{l}0,80 \pm 0,14 \\
0,95 \pm 0,19\end{array}$ & $\begin{array}{l}0,04 \pm 0,01 \\
0,03 \pm 0,01\end{array}$ \\
\hline Animaux à jeun & $\begin{array}{l}\text { Plasma ...... } \\
\text { Foie ........ }\end{array}$ & $\begin{array}{l}7,93 \pm 1,18 * \\
4,39 \pm 0,62 *\end{array}$ & $\begin{array}{l}0,34 \pm 0,06 * \\
0,45 \pm 0,07 *\end{array}$ \\
\hline
\end{tabular}

Les résultats correspondent à la moyenne \pm SEM et sont exprimés en $\mu$ moles $/ \mathrm{ml}$ d'eau plasmatique ou hépatique. La signification statistique pour $P<0,05$ est indiquée par *.

TABLEAU 4

Concentrations hépotiques de différents métabolites

\begin{tabular}{|c|c|c|c|c|c|}
\hline & Lactate & $\begin{array}{l}\text { 3-hydroxy- } \\
\text { butyrate }\end{array}$ & Acétoacétate & $\frac{3 \mathrm{OHB}}{A \subset A C}$ & Glycogène \\
\hline $\begin{array}{l}\text { Animaux nourris } \\
\text { Animaux à jeûn }\end{array}$ & $\begin{array}{l}2,08 \pm 0,07 \\
0,69 \pm 0,09 *\end{array}$ & $\begin{array}{l}0,65 \pm 0,13 \\
3,12 \pm 0,44 *\end{array}$ & $\begin{array}{l}0,02 \pm 0,01 \\
0,32 \pm 0,05 *\end{array}$ & $\begin{array}{l}32 \\
10\end{array}$ & $\begin{array}{r}28,46 \pm 4,08 \\
3,92 \pm 0,68 *\end{array}$ \\
\hline
\end{tabular}

Les valeurs exprimées en $\mu$ moles $/ g$ de poids frais sauf pour le glycogène $(\mathrm{mg} / \mathrm{g})$ correspondent à la moyenne $\pm S E M$ pour 11 cailles. La signification statistique pour $P<0,05$ est indiquée par *

\section{Discussion.}

A partir de ces résultats, ef en accord avec ceux obtenus par différents auteurs, on constate que la glycémie des oiseaux est très supérieure à celle mesurée chez les mammifères. De plus, les concentrations de glucose plasmatique sont plus élevées que celles du sang car les érythrocytes contiennent peu ou pas de glucose (Bell, 1971). Un jeûne de $72 \mathrm{~h}$ produit chez la caille comme chez le poulet (Langslow et al., 1970 ; Davison et Langslow, 1975), une baisse modérée mais significative de la glycémie. Ces variations de glycémie sont de faible amplitude. Chez la poule, Hazelwood et Lorenz (1959) signalent que lors d'un je ûne prolongé (6 jours) les quantités de glucose s'élèvent après le $3^{e}$ jour et deviennent supérieures à celles de l'état initial. Chez le poulet, Belo ef al. (1976) et Brady ef al. (1978) n'ont noté aucune variation de la glycémie après un jeûne comparable à celui utilisé dans nos expériences. Par rapporf aux résultats obtenus chez le rat, nos expériences sur la caille confirment donc le fait que les oiseaux maintiennent des taux de glucose élevés lors du jeûne.

En période de jeûne, la glycogénolyse hépatique n'est pas suffisante pour maintenir à elle seule la glycémie à un taux normal. D'après Hazelwood et Lorenz (1959), les stocks de glycogène sont épuisés après $24 \mathrm{~h}$ de jeûne chez le poulet et réaugmentent 
légèrement lorsque le jeûne est prolongé ; le glycogène est alors formé par néoglucogenèse. Cette voie permet donc de satisfaire aux besoins en glucose, on assiste à une augmentation de son activité. D'une façon générale, l'oiseau semble avoir une bonne aptitude à assurer ses propres besoins en glucose puisqu'il s'adapte bien à un régime dépourvu de glucides (Gauthier et al., 1979).

Chez le poulet, l'importance des différents substrats glucoformateurs a fait l'objet de nombreuses recherches. Les travaux de Sarkar (1971), Davison et Langslow (1975), Brady et al. (1979), Dickson et Langslow $(1977,1978)$ ont montré que le lactate est le principal substrat utilisé pour accroître la production de glucose. Le lactate est un substrat d'autant plus important que les tissus musculaires de l'oiseau sont particulièrement riches en muscles blancs dont les capacités de métabolisme aérobie sont faibles. C'est pourquoi l'utilisation du glucose chez l'oiseau est particulièrement élevée et il existe un recyclage du glucose en lactate très important (Belo et al., 1976). La chute des concentrations hépatiques et plasmatiques du lactate chez la caille à jeûn suggère que ce substrat est utilisé activement dans le processus de néoglucogenèse.

L'alanine joue un rôle essentiel dans la glucogenèse chez le rat et l'homme (cycle de Felig). Chez le poulet ou le pigeon, l'importance de l'alanine semble différente. Un jeûne de 24 h chez le poulef n'entraîne aucune baisse de l'alaninémie (Belo ef al., 1976). Ainsi, Sarkar (1971), Dickson et Langslow (1977), Brady ef al. (1979) ont montré que l'administration d'alanine à des poulets à jeûn, ou l'incubation d'hépatocytes isolés avec cel acide aminé, avaił peu d'influence sur la production de glucose. Néanmoins, Davison et Langslow (1975) ont observé une augmentation de la glycémie après injection d'alanine. Ces différences par rapport au rat peuvent s'expliquer par la localisation ou l'activité de certaines enzymes chez le poulet. Dans cette espèce, l'alanine aminotransférase est presque exclusivement localisée dans la mitochondrie et son activité est nettement plus faible que chez le rat (Sarkar, 1974, 1977), ce qui réduirait le taux de conversion de l'alanine en pyruvate. La localisation mitochondriale de la PEP carboxykinase, en réduisant la production de NADH dans le cytosol, liée au transport des composés à 4 atomes de carbone, pourrait contribuer à la faible utilisation de l'alanine.

La réponse au jeune chez la caille adulte est aussi caractérisée par une élévation des acides gras libres plasmatiques. Le même phénomène est observé chez le poulet (Langslow ef al., 1970 ; Lepkovsky et al., 1967). On observe aussi une chute du taux des triglycérides dans la mesure où les acides gras libres sont peu réestérifiés par le foie et où la sécrétion de lipoprotéines est diminuée. De plus, la lipogenèse hépatique diminue chez la caille à jeun pour réaugmenter après réalimentation (Shapira et al., 1979).

Les acides gras libres, libérés par le tissu adipeux, subissent au niveau du foie une $\beta$-oxydation car en période de jeûne, la synthèse des lipides diminue, que ce soit celle des triglycérides ou du cholestérol (Leveille, 1969). Il semble, chez l'oiseau comme chez le mammifère, qu'un déséquilibre dans la disponibilité en composés glucoformateurs et en acides gras libres, soit à l'origine des cétoses du jeûne ou de certains régimes hyperlipidiques lorsque l'apport protéique est insuffisant (Renner et Elcombe, 1967). Parmi les corps cétoniques, le 3-hydroxybutyrate est le composé majeur circulant, les taux d'acétoacétate sont toujours très faibles. Le rapport 3-hydroxybutyrate/acétoacétate est nettement plus élevé que celui observé chez le rat. Au niveau du 
foie, on constate qu'à jeun le 3-hydroxybutyrate produit est libéré dans le plasma apparemment contre un gradient de concentration, puisque les concentrations plasmatiques en 3-hydroxybutyrate sont presque deux fois plus élevées que dans le foie. Ceci avait été décrit précédemment chez le poulet à jeun (Brady et al., 1978). Un système de transport facilité ou actif des corps cétoniques à travers la membrane cellulaire doit donc être envisagé. De tels systèmes ont été décrits dans des études effectuées sur des érythrocytes et des thymocytes (Andersen ef al., 1978) ainsi qu'au niveau des bordures en brosse des membranes des tubules rénaux (Garcia ef al., 1980).

Le rapport 3-hydroxybutyrate/acétoacétate très élevé à jeun pose le problème de l'état redox mitochondrial. En effet, l'élévation du rapport NADH/NAD dans la mitochondrie des espèces possédant une PEPCK mitochondriale devrait théoriquement inhiber la formation du phosphoénol pyruvate. Or la production de glucose semble très efficace à jeun. Certains auteurs (Brady ef al., 1978) ont suggéré l'existence d'une anomalie dans le fonctionnement de la 3-hydroxybutyrate déshydrogénase. En fait, il semble qu'on puisse faire l'hypothèse nouvelle que c'est la vitesse de transport du 3-hydroxybutyrate hors de la mitochondrie et hors de la cellule qui est différente de celle de l'acétoacétate et qui permet l'exportation de NADH.

L'élévation des corps céłoniques sanguins pourrait aussi résulter d'une mauvaise utilisation par les tissus périphériques. Ceci a été constaté chez l'homme lors de je ûnes de longues durées : l'utilisation maximale des corps cétoniques par le muscle a lieu pour des concentrations voisines de $2 \mathrm{mM}$ (Garber et al., 1974). De plus, chez l'oiseau, l'activité de la 3-hydroxybutyrate déshydrogénase semble limitante pour l'utilisation du 3-hydroxybutyrate par les muscles de la poitrine (Bailey et Horne, 1972).

La diminution de la glycémie chez la caille à jeun s'accompagne d'une activation de la néoglucogenèse à partir du lactate et probablement de certains acides aminés, d'une libération d'acides gras libres et d'une augmentation de la synthèse des corps cétoniques. Ce travail montre donc que la réponse de la caille à jeun ne diffère pas particulièrement de celle des autres oiseaux. Toutefois, de nombreux problèmes subsistent au sujet de l'utilisation des corps cétoniques et du glucose par les tissus périphériques ef de l'utilisation de certains substrats pour la néoglucogenèse (alanine, pyruvate).

Reçu en juin 1980.

Accepté en décembre 1980.

\section{Références}

ANDERSEN B. L., TARPLEY H. L., REGEN D. M., 1978. Characterization of $\beta$-hydroxybutyrate transport in rat erythrocytes and thymocytes Biochim. biophys. Acta, 508, 525-528.

BAILEY E., HORNE J. A., 1972. Formation and utilization of acetoacetate and D-3-hydroxybutyrate by various tissues of the adult pigeon (Columbia livia). Comp. Biochem. Physiol., 42, 659-667.

BELL D. J., 1971. Plasma glucose. In BELL D. J., FREEDMAN B. M., Physiology and biochemistry of the domestic fowl, vol. 2, 913-919. Acad. Press, London.

BELO P. J., ROMSOS D. R., LEVEILLE G. A., 1976. Blood metabolites and glucose metabolism in the fed and fasted chicken. J. Nutr., 106, 1135-1143.

BERGMEYER H. U., BERNT E., SCHMIDT F., STORK H., 1974. Glucose : determination with hexokinase and glucose-6-phosphate dehydrogenase. In BERGMEYER H. U., Methods of enzymatic onalysis, vol. 3, 1196-1201. Acad. Press, Londres et N. Y. 
BRADY L. J., ROMSOS D. R., BRADY P. S., BERGEN W. G., LEVEILLE G. A., 1978. The effects of fasting on body composition, glucose furnover, enzymes and metabolites in the chicken. $J$. Nutr., 108, 648-657.

BRADY L. J., ROMSOS D. R., LEVEILLE G. A., 1979. Gluconeogenesis in isolated chicken (Gallus domesticus) liver cells. Comp. Biochem. Physiol., 63, 193-198.

DAVISON T., LANGSLOW D. R., 1975. Changes in plasma glucose and liver glycogen following the administration of gluconeogenic precursors to the starving fowl. Comp. Biochem. Physiol., 52, 645-649.

DICKSON A. J., LANGSLOW D. R., 1977. Gluconeogenesis in isolated chicken hepatocytes. Biochem. Soc. Trans., 5, 983-986.

DICKSON A. J., LANGSLOW D. R., 1978. Hepatic gluconeogenesis in chickens. Mol. cell. Biochem., 22, 167-181.

FAHLOLT K., LUND B., FAHLOLT W., 1973. An easy colorimetric micromethod for routine determination of free fatty acids in plasma. Clin. chim. Acta, 41, 105-111.

GARBER A. J., MENZEL P. H., BODEN G., OWEN O. E., 1974. Hepatic ketogenesis and gluconeogenesis in humans. J. clin. Invest., 54, 981-989.

GARCIA M. L., BENAVIDES J., VALDIVIESO F., 1980. Ketone body transport in renal brush border membrane vesicles. Biochim. biophys. Acta, 600, 922-930.

GAUTHIER A., LARBIER M., BLUM J. C., 1979. Influence des glucides alimentaires sur la croissance ef la vitesse d'absorption intestinale du glucose ef du fructose chez le poulef. Ann. Biol. anim., Bioch. Biophys., 19, 703-707.

GUTMANN I., WAHLEFELD A. W., 1974. $\mathrm{L}^{(+)}$-lactate determination with lactate dehydrogenase and NAD. In BERGMEYER H. U., Methods of enzymatic analysis, vol. 3, 1464-1468. Acad. Press, Londres et $N$. Y.

HAZELWOOD R. L., LORENZ F. W., 1959. Effects of fasting and insulin on carbohydrate metabolism of the domestic fowl. Am. J. Physiol., 197, 47-51.

KEPPLER D., DECKER K., 1974. Glycogen determination with amyloglucosidase. In BERGMEYER H. U., Methods of enzymatic analysis, Vol. 3, 1127-1131. Acad. Press, Londres et N. Y.

LANGSLOW D. R., BUTLER E. J., HALES C. N., PEARSON A. W., 1970. The response of plasma insulin, glucose and non-esterified fatty acids to various hormones, nutrients and drugs in the domestic fowl. J. Endocr., 46, 243-260.

LANGSLOW D. R., HALES C. N., 1971. The role of the endocrine pancreas and catecholamines in the control of carbohydrate and lipid metabolism. In BELL D. J., FREEMAN B. M., Physiology and biochemistry of the domestic fowl, vol. 1, 521-547. Acad. Press, London.

LARDY H. A., 1975. Gluconeogenesis pathways and hormonal regulation. Harvey Lect., 60, 261-278.

LEPKOVSKY S., DIMICK M. K., FURUTA F., SNAPIR N., PARK R., NARITA N., KOMATSU K., 1967. Response of blood glucose and plasma free fatty acids to fasting and to injection of insulin and testosterone in chickens. Endocrinology, 81, 1001-1006.

LEVEILLE G. A., 1969. In vivo fatty acid and cholesterol synthesis in fasted and fasted-refed chicks. J. Nutr., 98, 367-372.

MELLANBY J., WILLIAMSON D. H., 1974. Acetoacetate. In BERGMEYER H. U., Methods of enzymafic analysís, vol. 4, 1840-1843. Acad. Press, Londres et N. Y.

O'NEILL I. E., LANGSLOW D. R., 1978. Glucose phosphorylation and dephosphorylation in chicken liver. Comp. Biochem. Physiol., 59, 317-325.

PEARCE J., 1971. Carbohydrate metabolism in the domestic fowl. Proc. Nutr. Soc., 30, 254-259.

PEARCE J., 1977. Some differences between Avian and Mammalian biochemistry. Int. J. Biochem., 8, 269-275.

RENNER R., ELCOMBE A. M., 1967. Metabolic effects of feeding « carbohydrate-free » diets to ch icks. J. Nutr., 93, 31-36.

RÖSCHLAU V. P., BERN E., GRUBER W., 1974. Enzymatische Bestimmung des Gesamt-cholesterins im Serum. Z. Klin. Chem. Klin. Biochem., 12, 403-407.

SARKAR N. K., 1971. Gluconeogenesis and the factors that control the process in chickens. Life Sci., 10, 293-300.

SARKAR N. K., 1974. Alanine and aspartate aminotransferase activities in serum and various subcellular fractions from the livers of different species. Int. J. Biochem., 5, 375-381. 
SARKAR N. K., 1977. Implication of alanine and aspartate aminotransferase, glutamic dehydrogenase and phosphoenol pyruvate carboxykinase in glucose production in rats, chicken and pigs. Int. J. Biochem., 8, 427-432.

SHAPIRA N., NIR I., BUDOWSKI P., 1979. Response of lipogenic enzymes and plasma lipids to starvation and refeeding in the adult japanese quail (Coturnix coturnix japonica). Br. J. Nutr., 42, 437-443.

SÖLING H., KLEINEKE J., 1976. Species dependent regulation of hepatic gluconeogenesis in higher animals, 369-462. In HANSON R. W., MEHLMAN M. A., Gluconeogenesis. Its regulation in mammalian species. Wiley et Sons, New York.

SÖLING H., KLEINEKE J., WILLMS B., JANSON G., KUHN A., 1973. Relationship between intracellular distribution of phosphoenol pyruvate carboxykinase, regulation of gluconeogenesis and energy cost of glucose production. Eur. J. Biochem., 37, 233-243.

WAHLEFELD A. W., 1974. Triglycerides. Determination after enzymatic hydrolysis. In BERGMEYER H. U., Methods of enzymatic analysis, vol. 4, 1831-1835. Acad. Press, Londres et N. Y.

WILLIAMSON D. H., 1974. L-Alanine. In BERGMEYER H. U., Methods of enzymatic analysis, vol. 4, 1679-1685. Acad. Press, Londres ef N. Y.

WILLIAMSON D. H., MELLANBY J., 1974. D-(-)-3-hydroxybutyrate. In BERGMEYER H. U., Methods of enzymatic analysis, vol. 4, 1836-1838. Acad. Press, Londres et N. Y. 